\title{
Role of Posterior Ligamentous Reinforcement in Proximal Junctional Kyphosis: A Cadaveric Biomechanical Study
}

\author{
Jun Sup Kim ${ }^{1}$, Zoe Beatrice Cheung ${ }^{1}$, Varun Arvind ${ }^{1}$, John Caridi ${ }^{2}$, Samuel Kang-Wook Cho ${ }^{1}$ \\ ${ }^{1}$ Department of Orthopaedic Surgery, Icahn School of Medicine at Mount Sinai, New York, NY, USA \\ ${ }^{2}$ Department of Neurosurgery, Icahn School of Medicine at Mount Sinai, New York, NY, USA
}

\section{Study Design: Cadaveric biomechanical study.}

Purpose: The purpose of this study was to biomechanically evaluate the effect of preserving or augmenting the interspinous ligament (ISL) and supraspinous ligament (SSL; ISL/SSL) complex between the upper instrumented vertebra (UIV) and UIV+1 using a cadaveric model.

Overview of Literature: Adult spinal deformity is becoming an increasingly prevalent disorder, and proximal junctional kyphosis (PJK) is a well-known postoperative complication following long spinal fusion.

Methods: Pure moments of 4 and $8 \mathrm{Nm}$ were applied to the native and instrumented spine, respectively $(n=8)$. The test conditions included the following: native spine (T7-L2), fused spine (T10-L2), fused spine with a hand-tied suture loop through the spinous processes at T9-T10, and fused spine with severed T9-T10 ISL/SSL complex.

Results: The flexion range of motion (ROM) at T9-T10 of the fused spine loaded at $8 \mathrm{Nm}$ increased by $62 \%$ compared to that of the native spine loaded at $4 \mathrm{Nm}$. The average flexion ROM at T9-T10 for the suture loop and severed ISL/SSL spines were $141 \%(p=0.13)$ and $177 \%(p=0.66)$ of the native spine at $4 \mathrm{Nm}$, respectively ( $p$-values vs. fused).

Conclusions: Transection of the ISL/SSL complex did not significantly change flexion ROM at the proximal junctional segment following instrumented spinal fusion. Furthermore, augmentation of the posterior ligamentous tension band with a polyester fiber suture loop did not mitigate excessive flexion loads on the proximal junctional segment. We postulate that the role of the posterior ligamentous tension band in mitigating PJK is secondary to the anterior column support provided by the vertebral body and intervertebral disc.

Keywords: Scoliosis; Proximal junctional kyphosis; Adult spinal deformity; Cadaver; Biomechanics

\section{Introduction}

Adult spinal deformity is becoming an increasingly prevalent disorder as a result of an aging population. Long multilevel fusions are frequently required to address adult spinal deformity, and these surgeries are associated with high rates of complication and revision. Proximal junctional kyphosis (PJK) is a well-known postoperative complication following long spinal fusion for deformity and has considered much attention in recent studies. First defined by Glattes et al. [1], PJK is characterized by a proximal junctional sagittal Cobb angle at least $10^{\circ}$ greater than the

Received Apr 27, 2018; Revised Jun 10, 2018; Accepted Jun 27, 2018

Corresponding author: Samuel Kang-Wook Cho

Department of Orthopaedic Surgery, Icahn School of Medicine at Mount Sinai, 5th Floor, 425 West 59th Street, New York, NY 10019, USA

Tel: +1-212-241-0276, Fax: +1-212-636-3102,E-mail: Samuel.Cho@mountsinai.org

*Portions of this work were presented in abstract form at the 52nd Annual Scoliosis Research Meeting in Philadelphia, PA, USA. 
preoperative measurement. The incidence of PJK is typically between $20 \%$ and $40 \%$ [1-3].

Multiple risk factors have been associated with PJK, including increasing age [1,4-7], osteoporosis $[6,8,9]$, presence of other comorbidities [4,9], preoperative sagittal malalignment $[8,10-13]$, disruption of posterior soft tissues [1-4,14-17], combined anterior and posterior approach $[2,15,18]$, rigidity of the construct [19-21], choice of the upper instrumented vertebra (UIV) $[1,4,8,10,11,22]$, and magnitude of the correction [3,5,10-12,17]. Among these, disruption of posterior soft tissues, specifically the posterior tension band, has been proposed as an important modifiable risk factor [1-4,14-17]. Cadaveric and biomechanical studies have demonstrated the importance of maintaining the integrity of posterior structures involved in the exposure of the UIV to prevent the loss of proximal junctional segment flexion stiffness [19,23]. In particular, the supraspinous ligament (SSL) and interspinous ligament (ISL) may play an important role as a posterior tension band in stabilizing the proximal junctional segment during flexion by acting as a tether against hyperflexion $[23,24]$.

Past studies have examined the biomechanical effects of sectioning the ISL above a long instrumented spinal fusion [19,23], but only few studies have attempted to reconstitute the posterior ligamentous tension band and restore proximal junctional segment flexion stiffness [24]. The purpose of this study was to biomechanically assess and evaluate the effect of preserving or augmenting the ISL/SSL complex between the UIV and UIV+1 using a cadaveric model. We hypothesized that preservation or augmentation of the ISL/SSL complex between the UIV and UIV +1 would mitigate excessive flexion loads on the proximal junctional segment immediately following surgery.

\section{Materials and Methods}

\section{Specimens}

Institutional review board approval was not required for this study. Eight (five males, three females) adult freshfrozen human cadaveric thoracolumbar spines (T7-L2) were used in this study. The mean age of the specimens was $59 \pm 20$ years (range, 25-76 years). The mean bone mineral density (BMD) and $\mathrm{T}$ score measured from the dissected lumbar (L1-L4) spine using dual energy X-ray absorptiometry (Discovery W; Hologic Inc., Marlborough, MA, USA) were $-2.7 \pm 1.0$ (range, -4.1 to -1.4 ) and $0.78 \pm 0.13$ (range, $0.60-0.94$ ), respectively. Specimens were evaluated grossly and radiographically to ensure the absence of pathologies such as fracture, ankylosis, or malignancy. Specimens were stored frozen at $-20^{\circ} \mathrm{C}$ and thawed to room temperature at $20^{\circ} \mathrm{C}$ for preparation and testing.

\section{Specimen preparation}

Soft tissue was dissected from the specimens, taking care to preserve the osseoligamentous tissues and intervertebral discs. The end vertebrae, T7 and L2, were partially embedded in polymethyl methacrylate bone cement, leaving the ligaments and intervertebral discs exposed. Small screws were placed in the anterior cortex of each vertebral body, lateral to the anterior longitudinal ligament, for a rigid attachment of three-dimensional (3D) motiontracking markers.

\section{Instrumentation}

Instrumentation was performed by two senior authors (S.K.C. and J.M.C.). Bilateral pedicle screws were placed at each level between T10 and L2 and connected with 5.5 $\mathrm{mm}$ stainless steel rods (Vitality; Zimmer Biomet Spine, Broomfield, CO, USA). The ISL/SSL complex was severed between T9 and T10. A polyester fiber suture $(5 \mathrm{~mm}$ Mersilene tape; Ethicon, Somerville, NJ, USA) was used to reconstitute the ISL/SSL complex by tying the suture loop through holes drilled in the T9 and T10 spinous processes. The suture loop was manually tightened prior to tying the knot. C-arm radiographs were obtained from the fused specimens prior to and after suture loop fixation to ensure no change in the sagittal angle of T9-T10.

\section{Biomechanical testing}

Each specimen was tested under four conditions: native spine, fused T10-L2 spine, fused T10-L2 spine with a severed ISL/SSL complex, and fused T10-L2 spine with a polyester fiber suture loop between $\mathrm{T} 9$ and $\mathrm{T} 10$ to reconstitute the transected ISL/SSL complex (Fig. 1). Prior to testing, each specimen was preconditioned through three cycles of pure moment loading in flexion-extension (FE), lateral bending (LB), and axial rotation (AR) at $4 \mathrm{Nm}$ 

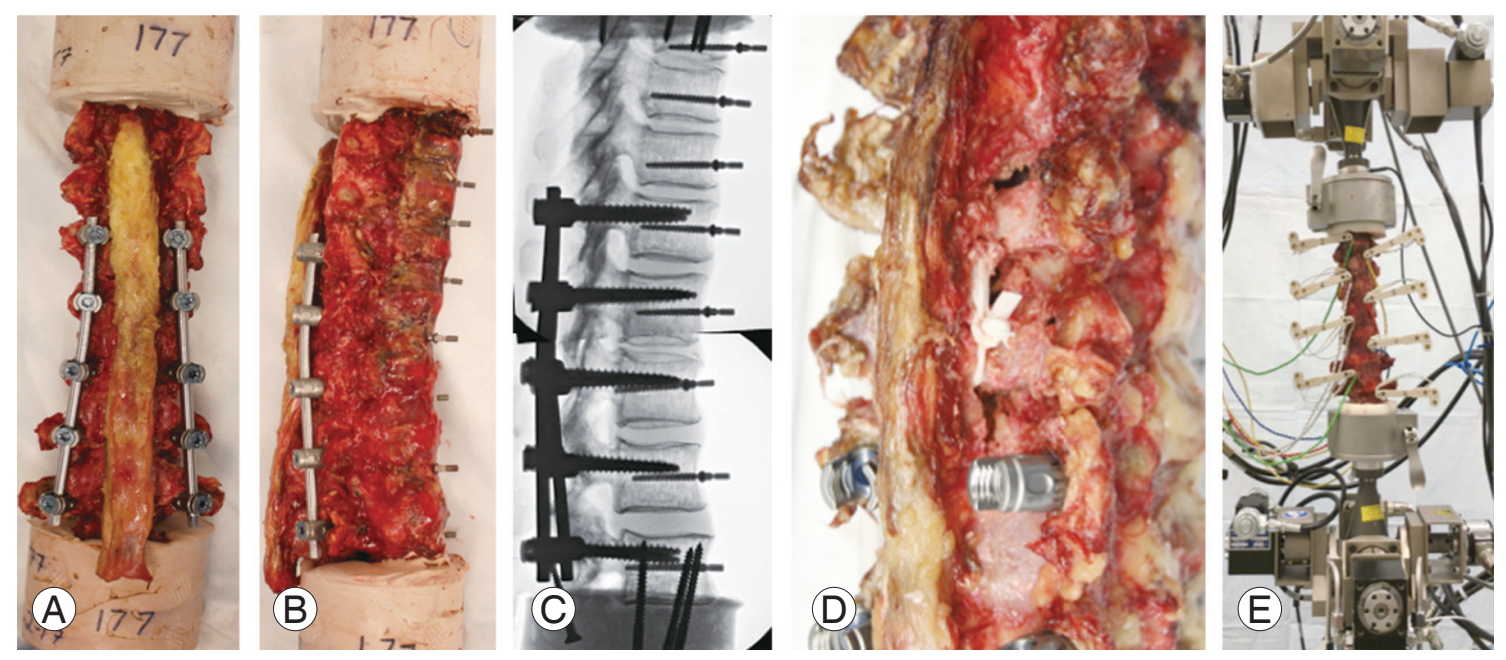

Fig. 1. Each thoracolumbar (T7-L2) specimen was prepared by partially potting the end vertebrae (T7 and L2), leaving the disc and ligaments exposed, and placing anterior screws to attach three-dimensional motion-tracking markers (A-C). Bilateral pedicle screws and rods were used for fusion fixation from T10 to $L 2$ (A, B). The superjacent segment (T9-T10) was bridged by a $5 \mathrm{~mm}$ hand-tied suture loop that passed through the T9 and T10 spinous processes (D). Biomechanical testing was performed using a servo-hydraulic axial/torsional test frame (MTS, Eden Prairie, MN, USA) with dual Bionix Spine Subsystem attachments (MTS) (E).

followed by three cycles at $8 \mathrm{Nm}$. Biomechanical testing was performed using a servo-hydraulic axial/torsional test frame (MTS, Eden Prairie, MN, USA) with dual Bionix Spine Kinematics Subsystem attachments (MTS). The subsystem was mounted onto a passive XY table to eliminate shear, and a $10 \mathrm{~N}$ axial compressive load was maintained throughout all testing to minimize tension. The native spine was loaded to $4 \mathrm{Nm}$ through three cycles in FE, LB, and AR. The fused spine (T10-L2), the fused spine with suture loop fixation at T9-T10, and the fused spine with severed ISL/SSL complex were loaded to $8 \mathrm{Nm}$ through three cycles in FE, LB, and AR, respectively. The $8 \mathrm{Nm}$ load represented the increased postoperative loads that could result from soft tissue dissection and decreased muscle function. The relative Euler angles were determined in flexion, FE, LB, and AR, respectively, using the MotionMonitor software (Innovative Sports Training Inc., Chicago, IL, USA), and the relative rotations of each motion segment were recorded using the Optotrak Certus 3D motion capture system (Northern Digital Inc., Waterloo, ON, Canada).

\section{Data analysis}

Range of motion (ROM) data from the third loading cycle in each direction (FE, LB, and $\mathrm{AR}$ ) were used for analysis. LOESS filtering with a smoothing factor of 0.01 was used to remove noise from the ROM-time data (R ver. 3.3.2, R-
Project; R Foundation for Statistical Computing, Vienna, Austria). The maximum ROM of each motion segment was then extracted and normalized to the ROM of the native spine loaded at $4 \mathrm{Nm}$. At the proximal junctional level (T9-T10), the ROM data for flexion, FE, LB, and AR were analyzed through two-way repeated-measures analysis of variance to compare the four different testing conditions. All data satisfied assumptions of normality according to the Shapiro-Wilk test. All statistical analyses were performed using Prism software (Prism ver. 7.01; GraphPad, La Jolla, CA, USA). Statistical significance was set at $p=0.05$.

\section{Results}

The ROM at the UIV/UIV+1 level abruptly increased from $33 \%$ of the native spine flexion ROM at T10-T11 to $162 \%$ of the native spine flexion ROM at T9-T10. FE ROM also increased abruptly from $28 \%$ of the native spine ROM at T10-T11 to $136 \%$ of the native spine ROM at T9-T10. Similarly, LB and AR ROM increased

Table 1. Change in lordosis angle compared go fused

\begin{tabular}{lcc} 
Intervention & Angle $\left({ }^{\circ}\right)$ & $p$-value \\
Suture loop & $0.2 \pm 0.2$ & $0.03^{\mathrm{a}}$ \\
\hline
\end{tabular}

Values are presented as mean \pm standard deviation.

a) Indicates that the angle change is significantly different from 0 . 
from $37 \%$ and $31 \%$ of the native spine ROM at T10-T11, respectively, to $132 \%$ and $128 \%$ of the native spine ROM at T9-T10, respectively. The mean change in lordosis at T9-T10 with the suture loop was $0.2^{\circ} \pm 0.2^{\circ}$. This change was significantly different from zero $(p<0.05)$ (Table 1$)$.

Figs. 2 and 3 summarize the results of the ROM biomechanical testing at T9-T10 under different testing conditions. There was no significant difference between the interventions and fused condition at T7-T8 and T8-T9.

The flexion ROM at T9-T10 in the fused spine loaded at $8 \mathrm{Nm}$ increased by $62 \%$ compared to the native spine loaded at $4 \mathrm{Nm}$. Flexion ROM was reduced from the fused state ( $162 \% \pm 31 \%$ of native spine) by the suture loop ( $141 \% \pm 31 \%$ of native spine). However, augmentation of the posterior ligamentous tension band with a polyester fiber suture loop did not significantly improve flexion ROM ( $p=0.13)$. When the ISL/SSL complex was transected, there was no significant change in the flexion ROM at T9-T10 ( $177 \% \pm 37 \%$ of native spine, $p=0.66)$. While the suture loop and cut ISL/SSL groups were not significantly different from the fused spine, the trends of change in flexion ROM were consistent across all specimens for suture loop (range, 6\%-43\% decrease in ROM) and cut ISL/ SSL complex (range, $0.6 \%-39 \%$ increase in ROM) compared to the fused spine (Table 2).

The FE ROM at T9-T10 in the fused spine loaded at $8 \mathrm{Nm}$ increased by $36 \%$ compared to the native spine loaded at $4 \mathrm{Nm}$. Reinforcing the posterior ligamentous complex with a polyester fiber suture loop reduced the FE ROM to $12 \%$ less than that of the fused spine with an intact ISL complex $(p=0.04)$. Severing the ISL/SSL complex significantly increased the FE ROM by $12 \%$ compared to the fused spine with an intact ISL complex $(p=0.04)$. FE ROM at T8-T9 with a severed ISL/SSL complex showed a $17 \%$ increase in ROM compared to the fused spine $(p=0.005)$.

The LB ROM at T9-T10 in the fused spine loaded at $8 \mathrm{Nm}$ increased by $32 \%$ compared to the native spine loaded at $4 \mathrm{Nm}$. There was no significant change after severing the ISL/SSL complex or augmenting with a polyester
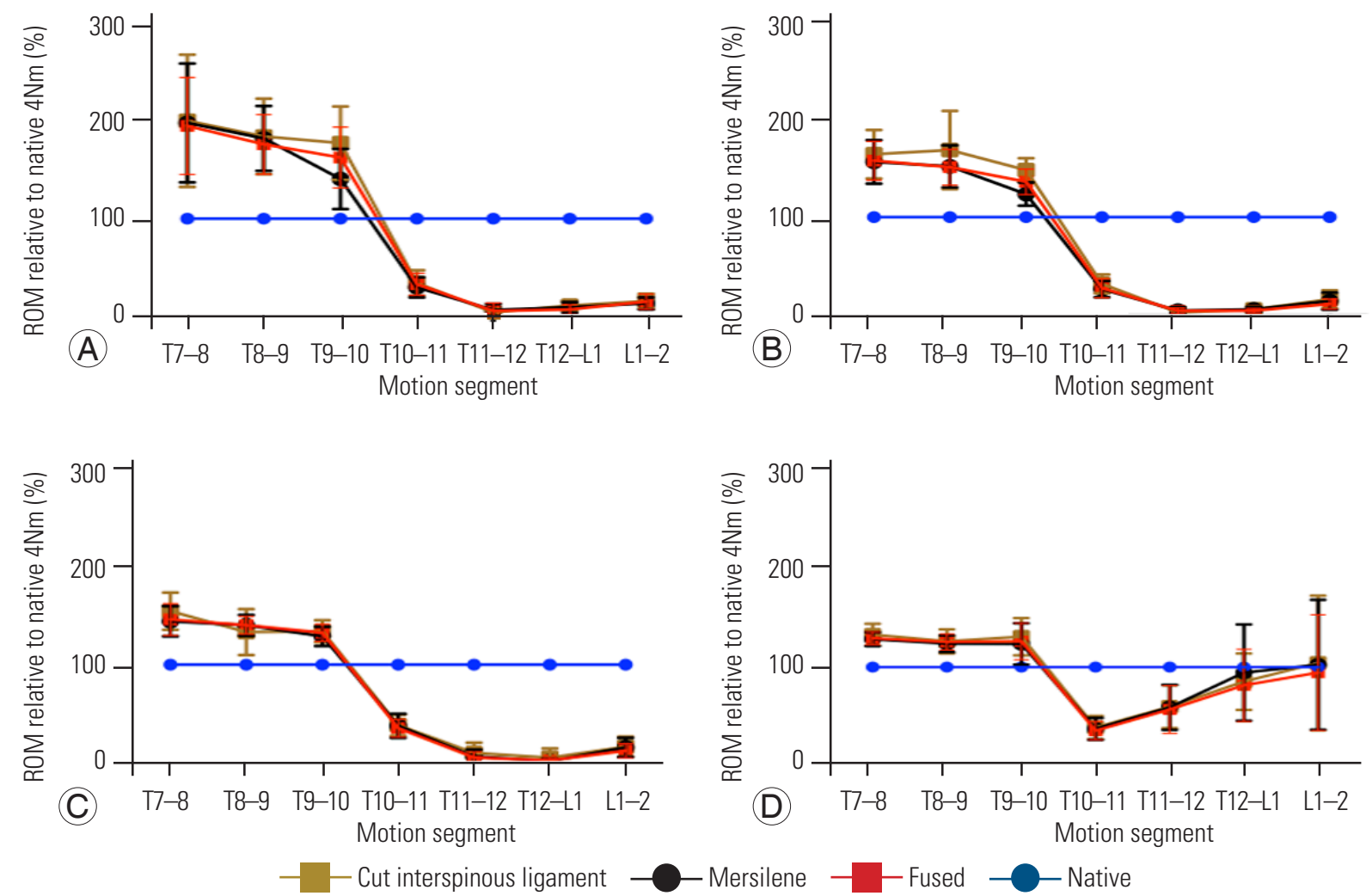

Fig. 2. ROM in flexion (A), flexion-extension (B), lateral bending (C), and axial rotation (D) across the thoracolumbar spine was largely unaffected, except at the index level (T9-T10), with each intervention technique. The native spine was loaded at $4 \mathrm{Nm}$ in each direction, while each instrumentation condition was loaded at $8 \mathrm{Nm}$ to simulate increased postoperative loading. Points represent mean, and error bars represent standard deviation. ROM, range of motion. 


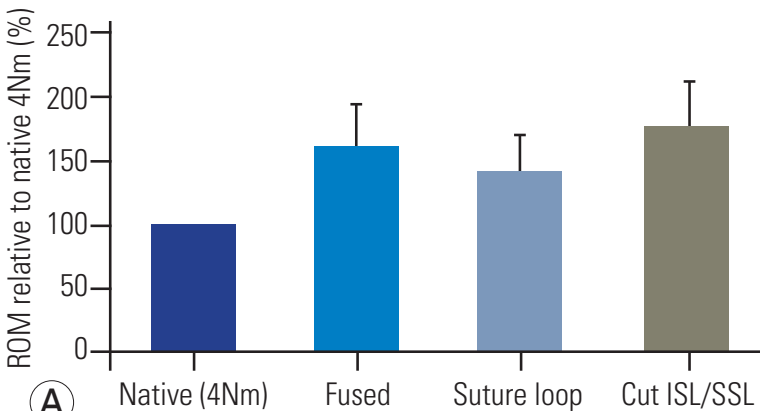

A Native (4Nm) Fused Suture loop Cut ISL/SSL

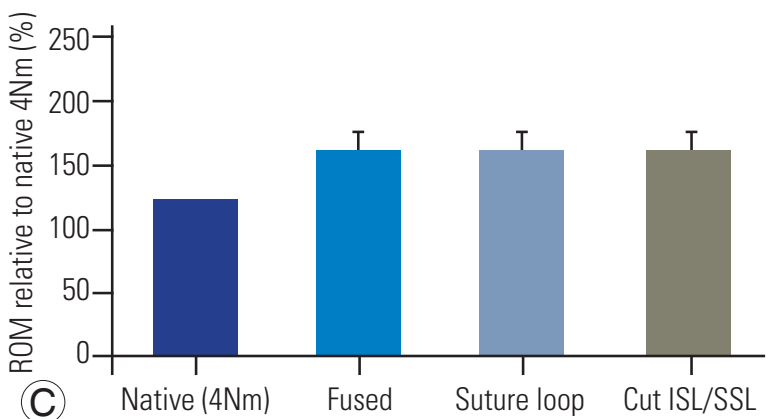

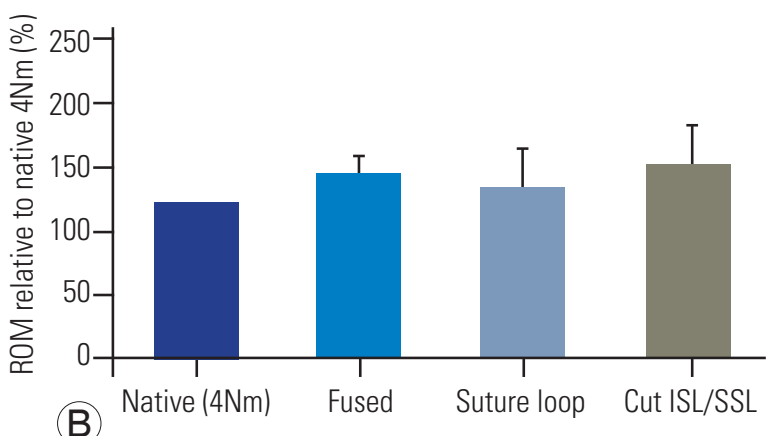

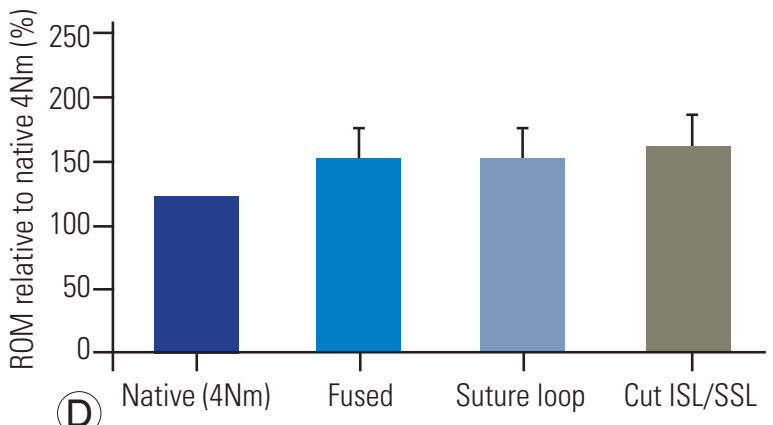

Fig. 3. Flexion range of motion (A) as well as extension (B) with minimal effects in lateral bending (C) and axial rotation (D) at the T9-T10 level. The native spine was loaded at $4 \mathrm{Nm}$ in each direction, while each instrumentation condition was loaded at 8 Nm to simulate increased postoperative loading. Bars represent mean, and error bars represent standard deviation. ROM, range of motion; ISL, interspinous ligament; SSL, supraspinous ligament.

Table 2. T9-10 flexion ROM compared to fused

\begin{tabular}{lcc} 
Intervention & $95 \%$ confidence interval & $p$-value \\
Suture loop & -2.868 to 46.20 & 0.1282 \\
Cut supraspinous ligament+interspinous ligament & -39.19 to 9.873 & 0.6609 \\
\hline
\end{tabular}

$p>0.05$ indicates that flexion ROM at upper instrumented vertebra+1 is not significantly different compared to fused.

$\mathrm{ROM}$, range of motion.

fiber suture loop ( $p=0.99)$. Similarly, the AR ROM at T9T10 in the fused spine loaded at $8 \mathrm{Nm}$ increased by $28 \%$ compared to the native spine loaded at $4 \mathrm{Nm}$, with no significant change after severing the ISL/SSL complex or reconstituting the posterior ligamentous complex with a polyester fiber suture loop ( $p=0.99$ ).

Notably, two cadaveric specimens failed during biomechanical testing secondary to fracture and were excluded from the analysis. Specimen LMD 16-519 (65-year-old female) had a lumbar BMD of 0.495 with an associated T score of -5.0. The other specimen that failed was LMD 15510 (69-year-old male) with a lumbar BMD of 0.898 and an associated $\mathrm{T}$ score of -1.8 . Of note, specimen LMD 371 (76-year-old female) with a lumbar BMD of 0.596 and an associated $\mathrm{T}$ score of -4.1 did not fail during biomechanical testing.

\section{Discussion}

PJK remains a significant postoperative complication following surgery for adult spinal deformity. While the pathophysiology of PJK is still not fully understood, it is likely to be multifactorial. One biomechanical theory is that $\mathrm{PJK}$ is due, in part, to an abrupt change in stiffness between the rigid instrumented vertebral segments and the relatively mobile proximal adjacent non-instrumented segments. This is consistent with the abrupt increase in flexion ROM at the UIV/UIV+1 level that is observed in our cadaveric model.

No definitive surgical techniques have been developed so far to prevent PJK. During posterior spinal fusions for adult spinal deformity, the ISL/SSL complex is usually released at most of the instrumented levels to increase 
flexibility and allow for more correction of the deformity. However, past studies have demonstrated the importance of preserving an intact ISL/SSL complex above a long spinal fusion $[19,23]$. At the proximal end of these constructs, many surgeons take precautions to preserve the ISL/SSL complex, but occasionally, it may be inadvertently sectioned at the proximal adjacent non-instrumented level.

This is the first human cadaveric biomechanical study to evaluate whether a surgical strategy to augment the posterior ligamentous tension band between the UIV and $\mathrm{UIV}+1$ with a polyester fiber suture could help mitigate the risk of PJK by alleviating excessive flexion loads on the proximal junctional segment following multilevel fusions. Biomechanical models of PJK assume that an increased load exists at the proximal segment following fusion, as is observed in our cadaveric model. The results of our study, however, demonstrate that transection of the ISL/SSL complex does not significantly change flexion ROM between the UIV and UIV+1. Furthermore, although subsequent augmentation of the posterior ligamentous tension band with a polyester fiber suture loop reduces overall FE ROM, it fails to improve excessive flexion ROM between the UIV and UIV+1. These results indicate that augmenting the posterior ligamentous complex with a fiber suture loop does not mitigate excessive flexion loads on the proximal junctional segment following instrumented spinal fusion. We suggest that the role of the posterior ligamentous tension band in mitigating PJK may only be secondary to anterior column support provided by the vertebral body and the intervertebral disc, explaining why clinical cases of PJK have significantly more wedging or compression fractures of the vertebral body than pure posterior distraction $[11,20]$. This notion is further supported by more recent data presented at the 52nd Annual Meeting of the Scoliosis Research Society in 2017 by Iyer et al. [25]. In a retrospective cohort study of 126 patients with 35 patients undergoing reinforcement of the posterior ligamentous structure using a nylon tape, they found no difference in the rate of PJK between the nylon tape cohort and the non-nylon tape cohort (26\% versus $28 \%$, $p=0.842$ ). Similarly, two cadaveric specimens were excluded from the current study secondary to a fracture of the vertebrae during biomechanical testing. Notably, both specimens had low T-scores, thereby suggesting the relative importance of BMD to the integrity of the posterior ligamentous structure in the development of PJK.
Our results differ from a recent study by Bess et al. [24]. Using a finite-element model, Bess et al. [24] investigated the ability of posterior anchored polyethylene tethers to distribute proximal motion segment stiffness in long instrumented spine constructs in the thoracolumbar spine. They demonstrated that augmentation of a long pedicle screw construct with an increasing number of posterior tethers created a more gradual transition from the instrumented to the non-instrumented spinal segments in ROM, pedicle screw loads, and forces in the posterior ligamentous complex, compared to constructs that used standard pedicle screw instrumentation or transverse process hooks at the UIV.

Several other biomechanical studies have also analyzed and compared the effects of different surgical strategies with the goal of reducing the risk of PJK. Anderson et al. [23] demonstrated, in a human cadaveric study, that soft tissue dissection done as part of the exposure and instrumentation of the UIV can reduce proximal adjacent motion segment stiffness. The single most important posterior structure that was likely to be disturbed by posterior instrumentation and arthrodesis was the ISL/SSL complex. Removal of the tethering effect of this ligamentous complex resulted in a $6.6 \%$ loss of flexion stability.

Cahill et al. [26] constructed a finite-element model of a thoracic spinal fusion to determine whether the use of transition rods could reduce the biomechanical risks of PJK. They first demonstrated that an intact ISL complex was integral in preventing abnormal disc pressure and angular displacement at levels above the UIV. Their results also demonstrated that the use of a transition rod at the $\mathrm{UIV}+1$ decreased the concentration of kyphosis-producing forces above the UIV that could potentially lead to PJK.

Cammarata et al. [19] used a computational model to analyze and compare the impact of the proximal dissection procedure, the implant type at the UIV, the sagittal rod curvature, and the proximal diameter of proximal transition rods on the biomechanical properties of the proximal junctional spinal segment. They demonstrated that the use of transverse process hooks at the UIV resulted in reduced biomechanical risk of PJK due to decreased rigidity at the UIV compared to the use of pedicle screws at the UIV. Their simulations showed similar results of decreased rigidity at the UIV with the use of tapered transition rods with reduced proximal diameters of $4 \mathrm{~mm}$ compared to the use of continuous $5.5 \mathrm{~mm}$ rods at the UIV. 
Lastly, they demonstrated that preservation of posterior structures at the UIV could also reduce the risk of PJK.

Similar to Cammarata et al. [19], Thawrani et al. [21] used a porcine thoracic spine model to show that transverse process hooks at the UIV resulted in a more gradual transition from rigid fixation to the normal mobility of the unfused spine in a long posterior spinal fusion construct compared to pedicle screws. All of the above biomechanical studies demonstrated that rigid fixation at the UIV creates a dramatic change in stiffness as the spine transitions from the instrumented to the non-instrumented spine. This difference in relative stiffness between the UIV and UIV +1 is thought to be a biomechanical risk factor for the development of PJK; thus, the occurrence of PJK may be reduced by less rigid constructs at the proximal terminus of the construct.

Limitations of this study include the length of the instrumented construct and the choice of the UIV. Our specimens were human cadaveric thoracolumbar spines, which included only the levels T7-L2. This limited both the upper and lower extent of our instrumented constructs. Past studies have demonstrated that proximal junctional failure occurs most often in the thoracolumbar region, with particularly high rates of failure when the UIV is at T10 $[8,10]$. However, our model may not simulate the biomechanical properties of longer multilevel constructs that extend up to the cervical spine and/or down to the sacropelvis. We were also limited in the choice of the UIV due to the nature of our cadaveric specimens. Our instrumentation ended at T10, but adult spinal deformity often requires long multilevel fusions that extend up to the cervical spine or cervicothoracic junction. Transitional areas have complex biomechanics; therefore, our results should be interpreted with caution for clinical cases of fusion that extend up to the cervicothoracic junction. We also do not consider the potential stabilizing effects of the costovertebral articulations and rib cage, although both have been shown in past studies to provide no significant contribution to motion segment flexion stiffness [27,28].

\section{Conclusions}

Our cadaveric biomechanical study demonstrates that transection of the ISL complex does not significantly change flexion ROM at the proximal junctional segment following instrumented spinal fusion. Furthermore, augmentation of the posterior ligamentous tension band with a polyester fiber suture loop does not mitigate excessive flexion loads on the proximal junctional segment. We postulate that the role of the posterior ligamentous tension band in mitigating PJK may only be secondary to anterior column support provided by the vertebral body and the intervertebral disc. This may explain why significantly more wedging or compression fractures of the vertebral body are seen in clinical cases of PJK than pure posterior distraction $[11,20]$. Future studies will need to more closely examine the interplay between the anterior column support and posterior ligamentous structures in mitigating excessive flexion loads on the proximal junctional segment. Alleviating this risk will be valuable in developing effective surgical techniques to prevent PJK.

\section{Conflict of Interest}

No potential conflict of interest relevant to this article was reported.

\section{Acknowledgments}

This study was funded by Zimmer Biomet Spine. Dr. Cho has financial relationships with Zimmer (paid consultant and research support), Globus (paid consultant), and Medtronic (paid consultant). Dr. Caridi has financial relationships with Zimmer (paid consultant and research support). None of the other co-authors have any financial relationships to disclose.

\section{Author Contributions}

Cadaveric dissections and biomechanical testing were performed by senior authors JMC and SKC. Statistical analyses and manuscript writing were performed by JSK, $\mathrm{ZBC}$, and VA.

\section{References}

1. Glattes RC, Bridwell KH, Lenke LG, Kim YJ, Rinella A, Edwards C 2nd. Proximal junctional kyphosis in adult spinal deformity following long instrumented posterior spinal fusion: incidence, outcomes, and risk factor analysis. Spine (Phila Pa 1976) 2005;30:1643-9.

2. Kim YJ, Bridwell KH, Lenke LG, Glattes CR, Rhim S, Cheh G. Proximal junctional kyphosis in adult spinal deformity after segmental posterior spinal instru- 
mentation and fusion: minimum five-year follow-up. Spine (Phila Pa 1976) 2008;33:2179-84.

3. Yagi $M$, King AB, Boachie-Adjei O. Incidence, risk factors, and natural course of proximal junctional kyphosis: surgical outcomes review of adult idiopathic scoliosis: minimum 5 years of follow-up. Spine (Phila Pa 1976) 2012;37:1479-89.

4. Bridwell KH, Lenke LG, Cho SK, et al. Proximal junctional kyphosis in primary adult deformity surgery: evaluation of 20 degrees as a critical angle. Neurosurgery 2013;72:899-906.

5. Kim HJ, Bridwell KH, Lenke LG, et al. Patients with proximal junctional kyphosis requiring revision surgery have higher postoperative lumbar lordosis and larger sagittal balance corrections. Spine (Phila Pa 1976) 2014;39:E576-80.

6. Kim HJ, Bridwell KH, Lenke LG, et al. Proximal junctional kyphosis results in inferior SRS pain subscores in adult deformity patients. Spine (Phila $\mathrm{Pa}$ 1976) 2013;38:896-901.

7. Kim HJ, Lenke LG, Shaffrey CI, Van Alstyne EM, Skelly AC. Proximal junctional kyphosis as a distinct form of adjacent segment pathology after spinal deformity surgery: a systematic review. Spine (Phila Pa 1976) 2012;37(22 Suppl):S144-64.

8. Hostin R, McCarthy I, O'Brien M, et al. Incidence, mode, and location of acute proximal junctional failures after surgical treatment of adult spinal deformity. Spine (Phila Pa 1976) 2013;38:1008-15.

9. Watanabe K, Lenke LG, Bridwell KH, Kim YJ, Koester L, Hensley M. Proximal junctional vertebral fracture in adults after spinal deformity surgery using pedicle screw constructs: analysis of morphological features. Spine (Phila Pa 1976) 2010;35:138-45.

10. Annis P, Lawrence BD, Spiker WR, et al. Predictive factors for acute proximal junctional failure after adult deformity surgery with upper instrumented vertebrae in the thoracolumbar spine. Evid Based Spine Care J 2014;5:160-2.

11. Maruo K, Ha Y, Inoue S, et al. Predictive factors for proximal junctional kyphosis in long fusions to the sacrum in adult spinal deformity. Spine (Phila Pa 1976) 2013;38:E1469-76.

12. Mendoza-Lattes S, Ries Z, Gao Y, Weinstein SL. Proximal junctional kyphosis in adult reconstructive spine surgery results from incomplete restoration of the lumbar lordosis relative to the magnitude of the thoracic kyphosis. Iowa Orthop J 2011;31:199-206.

13. Smith MW, Annis P, Lawrence BD, Daubs MD, Brodke DS. Early proximal junctional failure in patients with preoperative sagittal imbalance. Evid Based Spine Care J 2013;4:163-4.

14. Denis F, Sun EC, Winter RB. Incidence and risk factors for proximal and distal junctional kyphosis following surgical treatment for Scheuermann kyphosis: minimum five-year follow-up. Spine (Phila $\mathrm{Pa} 1976$ ) 2009;34:E729-34.

15. Kim HJ, Yagi M, Nyugen J, Cunningham ME, Boachie-Adjei O. Combined anterior-posterior surgery is the most important risk factor for developing proximal junctional kyphosis in idiopathic scoliosis. Clin Orthop Relat Res 2012;470:1633-9.

16. Kim YJ, Lenke LG, Bridwell KH, et al. Proximal junctional kyphosis in adolescent idiopathic scoliosis after 3 different types of posterior segmental spinal instrumentation and fusions: incidence and risk factor analysis of 410 cases. Spine (Phila $\mathrm{Pa}$ 1976) 2007;32:2731-8.

17. Yagi M, Rahm M, Gaines R, et al. Characterization and surgical outcomes of proximal junctional failure in surgically treated patients with adult spinal deformity. Spine (Phila Pa 1976) 2014;39:E607-14.

18. Wang J, Zhao Y, Shen B, Wang C, Li M. Risk factor analysis of proximal junctional kyphosis after posterior fusion in patients with idiopathic scoliosis. Injury 2010;41:415-20.

19. Cammarata M, Aubin CE, Wang X, Mac-Thiong JM. Biomechanical risk factors for proximal junctional kyphosis: a detailed numerical analysis of surgical instrumentation variables. Spine (Phila Pa 1976) 2014;39:E500-7.

20. Lau D, Clark AJ, Scheer JK, et al. Proximal junctional kyphosis and failure after spinal deformity surgery: a systematic review of the literature as a background to classification development. Spine (Phila Pa 1976) 2014;39:2093-102.

21. Thawrani DP, Glos DL, Coombs MT, Bylski-Austrow DI, Sturm PF. Transverse process hooks at upper instrumented vertebra provide more gradual motion transition than pedicle screws. Spine (Phila Pa 1976) 2014;39:E826-32.

22. Ha Y, Maruo K, Racine L, et al. Proximal junctional kyphosis and clinical outcomes in adult spinal deformity surgery with fusion from the thoracic spine 
to the sacrum: a comparison of proximal and distal upper instrumented vertebrae. J Neurosurg Spine 2013;19:360-9.

23. Anderson AL, McIff TE, Asher MA, Burton DC, Glattes RC. The effect of posterior thoracic spine anatomical structures on motion segment flexion stiffness. Spine (Phila Pa 1976) 2009;34:441-6.

24. Bess S, Harris JE, Turner AW, et al. The effect of posterior polyester tethers on the biomechanics of proximal junctional kyphosis: a finite element analysis. J Neurosurg Spine 2017;26:125-33.

25. Iyer S, Lovecchio F, Elysee JC, Lafage R, Schwab FJ, Kim HJ. Isolated posterior ligamentous reinforcement does not decrease proximal junctional kyphosis in adult spinal deformity. Proceedings of the Scoliosis Research Society 52nd Annual Meeting; 2017 Sep
6-9; Philadelphia, USA. Milwaukee (WI): Scoliosis Research Society; 2017.

26. Cahill PJ, Wang W, Asghar J, et al. The use of a transition rod may prevent proximal junctional kyphosis in the thoracic spine after scoliosis surgery: a finite element analysis. Spine (Phila Pa 1976) 2012;37:E68795.

27. Feiertag MA, Horton WC, Norman JT, Proctor FC, Hutton WC. The effect of different surgical releases on thoracic spinal motion: a cadaveric study. Spine (Phila Pa 1976) 1995;20:1604-11.

28. Oda I, Abumi K, Lu D, Shono Y, Kaneda K. Biomechanical role of the posterior elements, costovertebral joints, and rib cage in the stability of the thoracic spine. Spine (Phila Pa 1976) 1996;21:1423-9. 